\title{
Lumped parameter modeling of hybrid magnetomotive force electromechanical valve actuator
}

\author{
Muhammad Adnan Alvi ${ }^{1 *}$, Zhaohui Zhang ${ }^{1}$, Jawad Aslam² ${ }^{2}$ Saad $\mathrm{Ali}^{2}$, Emad Uddin ${ }^{2}$, Waqas Hassan \\ Tanveer $^{2}$, Syed Omar Gillani ${ }^{2}$, Ali Abbas Zaidi ${ }^{2}$ and Muhammad Yamin Younis ${ }^{3}$
}

\begin{abstract}
In this study, an innovative hybrid permanent magnet (PM)/electromagnet (EM) magneto-motive force (MMF) actuator is proposed for variable valve timing camless engines. The proposed design provides a large magnetic force with low energy consumption (startup and valve holding), PM demagnetization isolation, and improved transient response. The modified hybrid valve actuator (MHVA) was designed in ANSYS Maxwell software and validated empirically by prototype. A lumped parameter model (LPM) based on mathematical equations is developed to approximate Finite Element Method (FEM) and experimental results. The LPM is divided into electrical, magnetic and mechanical subsystems that are developed and integrated in MATLAB software. The comparative analysis of LPM, FEM and empirical results confirmed improved dynamic performance and energy consumption.
\end{abstract}

Second part title: Text for this section.

Keywords: Permanent magnet; Electromagnet; Magneto-motive force; Camless engine; Modified hybrid valve actuator; Finite element method; Lumped parameter model

\section{Introduction}

Optimal fuel efficiency and engine performance can be achieved by variation of valve timing with engine speed. The conventional camshaft engines have valve

\footnotetext{
${ }^{*}$ Correspondence: adnanalvi@xs.ustb.edu.cn ORCID ID:

https://orcid.org/0000-0001-9606-8568

${ }^{1}$ School of Automation and Electrical Engineering, University of Science and Technology Beijing, Beijing 100083, China.

Full list of author information is available at the end of the article
}

events that can be optimized for finite range at engine development stage to meet engine load and speed requirements [1]. Variable camshaft timing (VCT) mechanism showed $3.5 \%$ reduced average fuel consumption on all part-load conditions for port fuel injection [2].

Camless valve-train (CVT) can provide a practical substitute to the conventional camshaft engine valve system. Different types of valve actuators have been tested to achieve flexible variable timing including motorized, piezoelectric, electrohydraulic, electropneumatic and electromagnetic actuators. Electrohydraulic actuator requires energy for driving the pump resulting in additional load on the engine. Moreover, the effectiveness of hydraulic fluid is dependent on its temperature and the impurities. Application of hydraulic actuators in commercial engines is discouraged due to higher chances of flammability and cost issues [3], [4]. A piezoelectric valve actuator has inherent problem of hysteresis loss to the actuation system, which inhibits precise position control of the actuator [5]. A motorized radial actuator requires linkage mechanisms for conversion of rotary to linear motion for the valve [6]. Liu et al.

Another promising measure for improving the engine performance is the use of electromagnetic actuators equipped with mass spring system. Double solenoid valve actuators have been proposed and prototyped. Commercial application of double solenoid actuators is not implemented due to inherent problems of complexity, consumption, noise and wear. Linear electromagnetic motor (LEM) based valve-trains have improved controllability as compared to double solenoid actuators [7]. LEM valve-train requires high power for large force generation during valve operation. Moving coil LEM valve-train design was proposed by [8], [9] and [10] and motor based LEM valve-train prototype was proposed by [11]. However, the above mentioned LEM valve-trains suffered from shorter operational life, which does not make them viable option for long term operations in engines. Zhang et al.

Transition time of actuator is dependent on armature mass, spring constant and viscous damping co- 
efficient of single degree of freedom mass spring system. Conventional electromagnetic actuators require a continuous large current to exceed the spring force and latch the armature to the seating position [12]. Large current generation leads to high power consumption by the actuator. Power consumption can be effectively lowered by utilizing hybrid actuator comprising a permanent magnet and an electromagnet. The power consumption required to overcome the spring force and latching the armature at the seat, can be avoided by using a permanent magnet (PM). PM reluctance latches the armature without current requirement [7], [13] and [14]. A hybrid valve actuator (HVA) consisting a non-asymmetric armature and rectangular core has been studied [15], [16], [12], [17] and [18]. A rectangular core $\mathrm{HVA}$ is limited to linear motion of armature to achieve failure free valve actuation. Moreover, a rectangular core HVA is susceptible to wear due to absence of cylindrical motion and torsional vibrations. Mechanical wear during valve actuation can be reduced by implementing a symmetrical armature design [19]. The EM and PM flux are in series with each other, resulting in demagnetization of PM due to inverse current. In an axis symmetric HVA with PM and EM flux in series, the demagnetization is prevented by leaving air gaps in the actuator assembly [8], [9] and [20].

In this study a modified hybrid valve actuator is designed and validated using FEM analysis and experimentation. This study includes the MHVA design instead of conventional EM actuator. This innovative design includes a parallel combination of solenoids employed for controlling PM (NdFe35) and EM reluctance. The energy consumption has been reduced by the use of a PM for latching valve at open and close position. A lumped parameter model (LPM) of MHVA is developed to approximate FEM and experimental results. The model is based on a system of mathematical equations used by [21]. The set of equations used, incorporates a magnetic saturation effect. The experimental results of MHVA are used to determine physical parameters for modeling the actuator. The results of LPM are compared with experimental and FEM for validation of model.

\section{Design and FEM analysis}

\subsection{Basic design of the MHVA}

The MHVA design is bi-directional and axis-symmetric, consisting of stationary PM and parallel configuration of coils with reciprocating steel-1008 armature Figure 1. The two cylindrical copper solenoid coils result in reducing the coil inductance and the transient time by rapid rise in current rate[22] and [23]. The upper and lower solenoid coils have the same number of turns and a similar wire diameter leading to low coil resistance. The EM flux is directed parallel to PM flux. The PM reluctance lowers energy consumption of the actuator by latching the armature at the seating position without coil current. Two springs attached to the armature provides a restoring force during armature oscillation. The design and selection of the springs directly affects the dynamics of armature [16] and [20]. The soft iron yoke has three parts including a yoke, a yoke cover plate, and a yoke base plate. The air gaps between yoke parts provide detour to the EM flux, thus preventing PM demagnetization. At the seating position, armature is attracted to the steel former which generates flux due to effect of PM flux.

The working of MHVA is depicted in Figure 2. At the beginning of the process, PM reluctance latches the armature to the lower seating position Figure $2 \mathrm{a}$. The valve is open at lower seating position of the armature. The inverse excitation current recharges the coil generating a release flux and reduces the PM flux, until the PM force is less than the spring force, releasing the armature form lower seat Figure $2 \mathrm{~b}$. The armature moves towards the other seating position resulting in closing of the valve. The excitation current is removed as the armature crosses the equilibrium position Figure 2c. The charging of upper solenoid coils causes PM holding force to overcome the spring force and latches the armature at upper seating position Figure $2 \mathrm{~d}$. The coils are discharged after latching of the armature at upper seat and PM holding force latches the armature at the seat keeping the valve closed Figure 2e. The $\mathrm{PM}$ included in the design results in reducing the energy consumption, as PM force holds the armature at the seating positions without the need of coil excitations. An inverse current is required for recharging of the coils and reducing PM flux allowing spring force to release the armature from seating position.

\subsection{FEM force analysis}

The FEM analysis is performed on the proposed MHVA in ANSYS Maxwell. The simulations are performed to validate the proposed actuator design under defined operating conditions. The FEM analysis showed that the armature holding force depends on the armature dimensions, PM, yoke parts, and air gaps. The labelled diagram and dimensions of construction parameters of MHVA are shown in Figure 3 and Table 1.

PM reluctance generates a holding force for latching the armature to seating position, and inverse current is required to release armature from seat. FEM analysis at the seating position without including inverse current effect showed the concentration of flux in former upper region, armature and contact area between 
Table 1: Dimensions of modelled MHVA construction parameters.

\begin{tabular}{llll}
\hline Variables Description & Variables Symbol & Variables Value & Material \\
\hline Armature & $A_{\text {rad }} \times A_{w} \times A_{t h}$ & $3 \times 22 \times 5 \mathrm{~mm}$ & Steel-1008 \\
Former & $F_{\text {rad }} \times F_{H} \times F_{c t h} \times F_{t h}$ & $8 \times 58 \times 8 \times 4 \mathrm{~mm}$ & Iron \\
Permanent Magnet & $P M_{\text {rad }} \times P M_{H} \times P M_{t h}$ & $8 \times 50 \times 4 \mathrm{~mm}$ & NdFe35 \\
Inner Coil & $I C_{\text {rad }} \times I C_{t h}$ & $12 \times 14 \mathrm{~mm}$ & Copper \\
Outer Coil & $O C_{\text {rad }} \times O C_{t h}$ & $26 \times 14 \mathrm{~mm}$ & Copper \\
Yoke Cover Plate & $Y C P_{\text {rad }} \times Y C P_{w} \times Y C P_{t h}$ & $27.7 \times 17.5 \times 9 \mathrm{~mm}$ & Iron \\
Yoke & $Y_{\text {rad }} \times Y_{H} \times Y_{w}$ & $40.2 \times 55.5 \times 3 \mathrm{~mm}$ & Iron \\
Yoke Base Plate & $Y B P_{\text {rad }} \times Y B P_{w} \times Y B P_{t h}$ & $13 \times 27 \times 5 \mathrm{~mm}$ & Iron \\
\hline
\end{tabular}

them Figure 4a. At the two ends of steel former, the flux is oriented in opposite direction for inverse current generation to demagnetization of PM releasing the armature. The same condition also applies for yoke base plate with opposite flux at the ends. Yoke and yoke cover plate have parallel directed flux, leading to a magnified holding force. Coils excited with inverse current results in opposition and cancellation of PM flux by EM flux to release the armature as shown in Figure 4b. The flux at the two ends of the armature is oppositely directed, resulting in negligible holding force. The flux concentration is in the lower former region with oppositely directed flux at the ends. Flux observed in the yoke parts and lower former region, is in the direction of EM flux, due to contact of yoke parts with the coils. FEM analysis at equilibrium position with no inverse current results in concentration of flux in lower region of the former and is shown in Figure 4c.

The flux flows through armature; yoke cover plate and partially flows through yoke in same direction as it flows through former upper region. Oppositely oriented flux is observed in lower former region; lower yoke region and yoke base plate leading to minimization of holding force.

The hybrid magnetomotive force response is observed in the FEM analysis under static conditions for PM with 0 Aturn and at three constant ampere turn values of 500 Aturn, 1000 Aturn and 1500 Aturn for EM. The FEM static force response is shown in Figure 5 . A voltage pulse of magnitudes $12 \mathrm{~V}$ and $24 \mathrm{~V}$, with a step of $1.5 \mathrm{~ms}$, is used as an input signal. The input voltage of $24 \mathrm{~V}$ power source resulted in rise of the current by $64 \%$ with peak value of $29 \mathrm{~A}$ as compared to $12 \mathrm{~V}$ power source having peak value of $6.5 \mathrm{~A}$. The current signal for both $12 \mathrm{~V}$ and $24 \mathrm{~V}$ step inputs are shown in Figure 6.

\section{Experimentation}

The MHVA is fabricated and tested to validate the design and FEM simulations of actuator. The parallel combination of coils was wound to AWG Wiring Standard sizing chart 15 gauge copper wire with a diameter of $1.4 \mathrm{~mm}$, comprising 400 turns over a nylon bobbin. The overall resistance of the parallel coils was 0.35 $\Omega$. Two similar precompressed linear springs were employed with preload of $75.28 \mathrm{~N}$ to provide a smooth and fast transient response leading to reduction in energy consumption. Universal Tensile Machine was utilized to obtain the stress strain curve for the springs. The spring constant value was $18.82 \mathrm{~N} / \mathrm{mm}$ obtained by performing linear regression on stress strain curve obtained from testing on ultimate tensile testing machine and viscous damping coefficient with a value of 3.559 $\mathrm{Ns} / \mathrm{m}$ was calculated from parameter identification of free oscillating under damped system.

The electrical and mechanical parameters of MHVA prototype are given in Table 2. The assembly of the hybrid actuator was fastened to a static test rig in a way that armature displacement was in vertical direction Figure 7. Opener/closer valve was tested for static experimentation. and for dynamic testing both the solenoids were used. A BUFSON load cell BSLZ1 , attached to the lower load cell plate was used to detect holding force. An HZ-891XL eddy current sensor (Shanghai Aerospace Vibration Instruments Co. Ltd., China), having a probe with power and amplification unit was utilized for the accurate position measurement of the armature. A thin sheet of $50 \mathrm{~mm}$ diameter and $1 \mathrm{~mm}$ thickness made of tin was used as a metal target. The power supplies were utilized in parallel and series combinations for various current and voltage requirements. The output voltage and current values achieved were $12 \mathrm{~V}$ and $24 \mathrm{~V}$ with $50 \mathrm{~A}$ current controlled with alteration of pulse width modulation (PWM). The current supplied to the MHVA was measured with a Hall-effect current sensor LA55P (LEM, Switzerland). The steady state force requirement was achieved by controlling MHVA current excitation. The MHVA was driven by integrated circuit (BTN7971B) consisting of half bridge configuration (Shenzhen Chipskey Technology Co., Ltd., China). The IC has a high current 60A, with maximum input voltage of $45 \mathrm{~V}$. The H-bride circuit was integrated 
with data acquisition of the actuator. The data acquisition (DAQ) and input/output control of MHVA was executed by National Instruments (NI) USB 6351. The NI LabVIEW software was utilized to program NI 6351 with a visual layout.

Table 2: Parameters of prototyped MHVA.

\begin{tabular}{ll}
\hline Overall Coil Resistance & $0.35 \Omega$ \\
Number of turns per coil (coil1/Coil2) & 200 \\
Moving Mass & $130 \mathrm{~g}$ \\
Spring Constant (k) & $18.82 \mathrm{~N} / \mathrm{mm}$ \\
Viscous Damping Coefficient (c) & $3.559 \mathrm{Ns} / \mathrm{m}$ \\
\hline
\end{tabular}

Armature was displaced using the lead screw for the analysis of holding force for each position at various values of constant current. An average of thousand values of force at each displacement was taken, varied from $0.5-4 \mathrm{~mm}$ to achieve reasonable precision. The force sensor was powered by 220VAC. The sensors including power module, position sensor, and current sensors were powered by a DC power supply. The analog data obtained from different sensors was logged in computer using data acquisition system. The variation of holding force with air gap observed during experimentation is plotted in the Figure 7. The force response at smaller air gaps becomes erroneous due to deflection of the armature, which is assumed rigid. The force at air gaps lower than $0.5 \mathrm{~mm}$ is not included to avoid this error. The experimental force behavior is reasonably close to the FEM force behavior Figure 5 and Figure 8. The current transient response was obtained for step input voltage signal of $12 \mathrm{~V}$ and $24 \mathrm{~V}$ without current control for the actuator. Quick rise in the current was observed for larger air gaps having low inductance value of magnetic circuit. For limiting the current magnitude, the duration of input voltage step was set to be $1.5 \mathrm{~ms}$ for large displacement of armature. The coil current signal transient response observed during experimentation is plotted in the Figure 9 .

The inverse current is required to reduce the PM reluctance to de-latch the armature from the seating position. The simulated and experimental inverse current is plotted with armature latched at the seat Figure 10. The maximum PM flux reduction is observed for 1500Aturn showing the holding force of $17.7 \mathrm{~N}$ for experimental and $13.92 \mathrm{~N}$ for simulated data. Error between experimental and FEM result is $3.7 \mathrm{~N}$. Increasing the Aturn value from 1500 Aturns tends to increase the holding force due to large flux generated by inverse coil current compared to PM flux which starts to attract the armature. This is the reason for restricting the experimental plot to 2000Aturns of inner/outer coil.
The force sensor, lead screw and load cell holding bolt were removed for free oscillation of the armature assembly. Initially the armature was kept at equilibrium position with help of precompressed spring and lead screw. The dynamic experimentation included releasing or catching of armature at one time for valve opening or closing Figure 11. The most important parameters for the dynamic testing included physical property values at low air gap influencing the landing velocity of armature. For the dynamic analysis, the feedback loop of position sensor and current sensor was required. For dynamic testing, pairs of coils are separately triggered by power driver circuit.

The power consumption for MHVA is considered for $12 \mathrm{~V} 7.5 \mathrm{~A}$. The power required by MHVA is to attract the armature for opening and closing of valve. The power consumed by MHVA remains constant as excitation current is required for latching the armature and an inverse excitation current of equal magnitude is used to de-latch the armature Table 4. PM reluctance provides holding force at the armature seat and power consumption is zero at the seating position.

Table 3: Energy consumption of MHVA.

\begin{tabular}{lll}
\hline Camshaft Speed $(\mathrm{rpm})$ & Time $(\mathrm{ms})$ & MHVA Energy $(\mathrm{J})$ \\
\hline $1000 \mathrm{rpm}$ & 60 & 0.36 \\
$2000 \mathrm{rpm}$ & 30 & 0.36 \\
$3000 \mathrm{rpm}$ & 20 & 0.36 \\
\hline
\end{tabular}

\section{Lumped Parameter Model}

Lumped parameter modeling is an effective methodology when modeling a multi-physic system. LPM investigates the effects of external parameters on the system performance without computation of FEA. A mathematical model of hybrid camless actuator system is developed based on set of mathematical equations [21].

Least square curve fitting was also performed on the experimental force data using a non-linear system of equations to verify the accuracy of developed LPM. This system of equation includes the magnetic saturation effects leading to an improved model as compared to the simplified linear system used to develop model in this study. The saturation effects incorporated in this system leads to a better control implementation. Non-linearities of flux linkage are mathematically represented by 1 .

$$
\lambda\left(x, i_{c}\right)=\lambda_{s}\left(1-e^{-i_{c} f(x)}\right)
$$

Where $\lambda$ is flux linkage of the coil, $\lambda_{s}$ is the saturated coil flux linkage and $i_{c}$ is the coil current. The function 
$\mathrm{f}(\mathrm{x})$ in 2 is used to relate varying armature position and is given in 2 .

$$
f(x)=\left(2 C_{1}\right) /\left(\left(C_{2}-x\right)\right)+C_{3}
$$

The function $\mathrm{f}(\mathrm{x})$ quantifies the severity level of saturation and $\lambda_{s}$ gives flux linkage values at saturation. The electromagnetic force can be derived from co-energy function given in 3 .

$$
W_{c}\left(x, i_{c}\right)=\int_{0}^{i_{c}} \lambda(x, \xi) d \xi
$$

Differentiation of co-energy function gives following expression.

$$
F\left(x, i_{c}\right)=\frac{\partial W_{c}\left(x, i_{c}\right)}{\partial x}
$$

The electromagnetic force expression is obtained by substituting 1 into 4 .

$$
\left.F\left(x, i_{c}\right)=\frac{\lambda_{s} f^{\prime}(x)}{f^{2}(x)}\left[1-\left[1+i_{c} f(x)\right] e^{(}-i_{c} f(x)\right)\right]
$$

Here $f^{\prime}(x)$ is given by the following expression.

$$
f^{\prime}(x)=\frac{2 C_{1}}{\left(C_{2}-x\right)^{2}}
$$

The parameters $\lambda_{s}, C_{1}, C_{2}$ and $C_{3}$ are determined by least square fitting of experimental force data under

\begin{tabular}{|c|c|}
\hline$\lambda_{s} \mathrm{rpm}$ & $0.054 \mathrm{~Wb}$ \\
\hline$C_{1} \mathrm{rpm}$ & $0.0065 \mathrm{~mm} / \mathrm{A}$ \\
\hline$C_{2} \mathrm{rpm}$ & $0.024 \mathrm{~mm}$ \\
\hline$C_{3} \mathrm{rpm}$ & $0.000218 A^{-1}$ \\
\hline
\end{tabular}
constraints. The curve fitting and the optimized parameter values obtained for all the data sets are given in Figure 12 and Table 3 respectively.

Table 4: Identified physical parameters from fitting.

The coil dynamic equation derived with saturation effects for non-linear system used by [21] is given in 7 . The non-linear system of equation with four fitted parameters is also used to develop LPM in a similar manner as the relatively simple system of equation with two fitted parameters. Eddy current effect is neglected using nonlinear system for LPM development.

$$
\frac{d i_{c}}{d t}=\frac{e^{i_{c} f(x)}}{\lambda f(x)}\left[v_{c}-R_{c} i_{c}-\lambda_{s} f^{\prime}(x) \dot{x} i_{c} e^{-i_{c} f(x)}\right]
$$

Step input voltage signals of $12 \mathrm{~V}$ and $24 \mathrm{~V}$ magnitude are used in the LPM development Figure 13. The LPM current peak shows $11 \%$ and $2 \%$ error compared with FEM and experimental peaks at $12 \mathrm{~V}$ Figure 14 . The error observed is $6 \%$ and $4 \%$ for LPM current peak at $24 \mathrm{~V}$ Figure 15. Lower peak values are obtained for smaller air gaps due to higher inductance values. The difference of current values for FEM and experimentation is due to voltage drop across the power drive board BTN7971B IC, line losses and limited power supplied by the power source. The drop observed for $24 \mathrm{~V}$ and $12 \mathrm{~V}$ power supply is $2 \mathrm{~V}$ and $0.98 \mathrm{~V}$ respectively. LPM is modelled using the experimental force data which leads to smaller error between LPM and experimental current peaks. The variation of LPM with FEM can be explained with simplified model neglecting eddy current and flux leakage effects.

The modeled force response gives comparable results with static experimental and FEM force behaviors. The LPM peak force at $24 \mathrm{~V}$ for $0.5 \mathrm{~mm}$ air gap gives $3 \%$ and $7 \%$ error with experimental and FEM results. The force response is shown in Figure 16. The comparison of LPM with experimentation and FEM results validate the LPM developed in this study.

The armature dynamics is modeled with single degree of freedom as mass spring damping system. The mechanical subsystem is developed using differential equation representing the forced and damped armature spring system is given by 8 .

$$
m \ddot{x}-c \dot{x}-k x=F\left(x, i_{c}\right)
$$

The armature displacement response is shown in Figure 17. The armature oscillates between fixed span of $8 \mathrm{~mm}$. The armature displacement starts at $-4 \mathrm{~mm}$ for valve opening and reaches the other extreme position. The armature displacement response for valve closing is similar due to symmetrical design of the actuator. The armature velocity reaches peak value of $1.82 \mathrm{~m} / \mathrm{s}$ at the equilibrium position Figure 18. The velocity response is dependent on current and force response. The velocity near the seating position can be controlled by the current response, to minimize stress and noise.

\section{Conclusion}

A novel hybrid valve actuator is analyzed in this study using FEM, prototype experimentation and LPM development. The valve actuator comprises of both PM 
and EM for force generation required for armature reciprocation and latching at the seating position. The use of PM in addition to EM in the actuator results in lowering the energy consumption. The physical parameters are identified using curve fitting on experimental data. The parameters are used in current and force equations to model corresponding behaviors. The force response was used to model the armature dynamics. The results of LPM are in good agreement with experimental and FEM results. The LPM can be used in control design to improve soft landing of valve.

\section{Acknowledgements}

The reseach is carried out for USTB and NUST and the authors sincerely thank to them for their support.

\section{Author's contributions}

MAA and JA conceived the idea of the study, wrote the manuscript and experiment; $Z Z$ were in charge and supervised the whole trial; SA, ED and WHT refined the ideas; SOG and AAZ contributed to structure design; MYY assisted with data analyses. All authors read and approved the final manuscript.

\section{Funding}

Not Applicable.

\section{Competing interests}

The authors declare that they have no competing interests.

\section{Author details}

${ }^{1}$ School of Automation and Electrical Engineering, University of Science and Technology Beijing, Beijing 100083, China.

${ }^{2}$ School of Mechanical and Manufacturing Engineering. Nationa University of Science and Technology, Islamabad, 44000, Pakistan.

${ }^{3}$ Department of Mechanical Engineering, Mirpur University of Science and Technology, Mirpur, 10250 (AJK), Pakistan

\section{References}

1. Hara, S., Suga, S., Watanabe, S., Nakamura, M.: Variable valve actuation systems for environmentally friendly engines. Hitachi Review 58(7), 319-324 (2009)

2. Bonatesta, F., Altamore, G., Kalsi, J., Cary, M.: Fuel economy analysis of part-load variable camshaft timing strategies in two modern small-capacity spark ignition engines. Applied Energy 164, 475-491 (2016)

3. Sun, Z., Cleary, D.: Dynamics and Control of an Electro-Hydraulic Fully Flexible Valve Actuation System. Proceedings of the American Control Conference 4, 3119-3124 (2003)

4. Sun, Z., Kuo, T.W.: Transient control of electro-hydraulic fully flexible engine valve actuation system. IEEE Transactions on Control Systems Technology 18(3), 613-621 (2010)

5. Jalili, N., Wagner, J., Dadfarnia, M.: A piezoelectric driven ratchet actuator mechanism with application to automotive engine valves. Mechatronics 13(8-9 SPEC.), 933-956 (2003)

6. Fabbrini, A., Garulli, A., Mercorelli, P.: A trajectory generation algorithm for optimal consumption in electromagnetic actuators. IEEE Transactions on Control Systems Technology 20(4), 1025-1032 (2012)

7. Liu, J.-J., Yang, Y.-P., Xu, J.-H.: Electromechanical Valve Actuator with Hybrid MMF for Camless Engine vol. 41, pp. 10698-10703. IFAC, ??? (2008)

8. Liu, L., Chang, S.: Improvement of valve seating performance of engine's electromagnetic valvetrain. Mechatronics 21(7), 1234-1238 (2011)

9. Liu, L., Chang, S.: Motion control of an electromagnetic valve actuator based on the inverse system method. Proceedings of the Institution of Mechanical Engineers, Part D: Journal of Automobile Engineering 226(1), 85-93 (2012)
10. Shao, D., Sichuan, X., Du, A.: Research on a New Electromagnetic Valve Actuator Based on Voice Coil Motor for Automobile Engines. SAE Technical Papers 2017-March(March) (2017)

11. Mercorelli, P.: A Two-Stage Sliding-Mode High-Gain Observer to Reduce Uncertainties and Disturbances Effects for Sensorless Control in Automotive Applications. IEEE Transactions on Industrial Electronics 62(9), 5929-5940 (2015)

12. Kim, J., Lieu, D.K.: A new electromagnetic engine valve actuator with less energy consumption for variable valve timing. Journal of Mechanical Science and Technology 21(4), 602-606 (2007)

13. Shiao, Y., Dat, L.V.: Actuator control for a new hybrid electromagnetic valvetrain in spark ignition engines. Proceedings of the Institution of Mechanical Engineers, Part D: Journal of Automobile Engineering 227(6), 789-799 (2013)

14. Lee, S.H., Yi, H.C., Han, K., Kim, J.H.: Genetic algorithm-based design optimization of electromagnetic valve actuators in combustion engines. Energies 8(11), 13222-13230 (2015)

15. Clark, R.E., Jewell, G.W., Forrest, S.J., Rens, J., Maerky, C.: Design features for enhancing the performance of electromagnetic valve actuation systems. IEEE Transactions on Magnetics 41(3), 1163-1168 (2005)

16. Kim, J., Lieu, D.K.: Designs for a new, quick-response, latching electromagnetic valve. 2005 IEEE International Conference on Electric Machines and Drives, 1773-1779 (2005)

17. Kim, J., Chang, J.: A new electromagnetic linear actuator for quick latching. 12th Biennial IEEE Conference on Electromagnetic Field Computation, CEFC 2006, 891676 (2006)

18. Rens, J., Clark, R.E., Jewell, G.W.: Static performance of a polarized permanent-magnet reluctance actuator for internal combustion engine valve actuation. IEEE Transactions on Magnetics 42(8), 2063-2070 (2006)

19. Albert, J., Banucu, R., Hafla, W., Rucker, W.M.: Simulation Based Development of a Valve Actuator for Alternative Drives Using BEM-FEM Code. IEEE Transactions on Magnetics 45(3), 1744-1747 (2009)

20. Shiao, Y., Dat, L.V.: A new electromagnetic valve train with $P M / E M$ actuator in SI engines. Transactions of the Canadian Society for Mechanical Engineering 37(3), 787-796 (2013)

21. Chung, S.K., Koch, C.R., Lynch, A.F..: Flatness-Based Feedback Control of an Automotive Solenoid Valve. IEEE Transactions on Control Systems Technology 15(2), 394-401 (2007)

22. Cope, D., Wright, A.: Electromagnetic fully flexible valve actuator SAE Technical Papers 2006(724) (2006)

23. Vu, D.: A Novel of Hybrid Magnet Engine Valve Actuator Using Shorted Turn for Fast Initial Response. International Journal of Information and Electronics Engineering 3(1), 95-98 (2013)

\section{Figures}

Figure 1: Assembled model of MHVA.

Figure 2: Step by step working of MHVA.

Figure 3: Construction parameters of proposed MHVA. 
Figure 4: Flux densities at the extreme position (no inverse current) (a), extreme position (with inverse current) (b), and equilibrium position (no inverse current) (c).

Figure 5: FEM Analysis of magnetomotive force response.

Figure 6: FEM Coil current transient responses at $12 \mathrm{~V}$ and 24.

Figure 7: Static test rig.

Figure 8: Experimental Force response (PM and EM).

Figure 9: Experimental coil current responses at $12 \mathrm{~V}$ and $24 \mathrm{~V}$.

Figure 10: Inverse coil current effect on holding force.

Figure 11: Dynamic test rig.

Figure 12: Curve fitting of Static Experimental Force Data.

Figure 13: Input voltage Signals for LPM.

Figure 14: Comparison of current response at $12 \mathrm{~V}$ and $0.5 \mathrm{~mm}$ (LPM, exp and FEM).

Figure 15: Comparison of current response at 24V and 0.5mm (LPM, exp and FEM).

Figure 16: LPM Force response at $\mathbf{0 . 5 m m}$ with $24 \mathrm{~V}$ input signal.
Figure 17: LPM armature displacement response.
Figure 18: LPM armature velocity response. 